\title{
DIFFERENTIAL POLYNOMIALS GENERATED BY SOME COMPLEX LINEAR DIFFERENTIAL EQUATIONS WITH MEROMORPHIC COEFFICIENTS
}

\author{
Benharrat Belä̈di AND AbDallah El FARissi \\ University of Mostaganem, Algeria
}

\begin{abstract}
In this paper, we investigate the relationship between small functions and differential polynomials $g(z)=d_{2} f^{\prime \prime}+d_{1} f^{\prime}+d_{0} f$, where $d_{0}(z), d_{1}(z), d_{2}(z)$ are meromorphic functions which are not all equal to zero with $\rho\left(d_{j}\right)<n(j=0,1,2)$ generated by some second order linear differential equations with meromorphic coefficients.
\end{abstract}

\section{INTRODUCTION AND STATEMENT OF RESULT}

Throughout this paper, we assume that the reader is familiar with the fundamental results and the standard notations of the Nevanlinna's value distribution theory (see $[8,10])$. In addition, we will use $\lambda(f)$ and $\lambda(1 / f)$ to denote respectively the exponents of convergence of the zero-sequence and the pole-sequence of a meromorphic function $f, \rho(f)$ to denote the order of growth of $f, \bar{\lambda}(f)$ and $\bar{\lambda}(1 / f)$ to denote respectively the exponents of convergence of the sequence of distinct zeros and distinct poles of $f$.

Consider the second order linear differential equation

$$
f^{\prime \prime}+A_{1}(z) e^{P(z)} f^{\prime}+A_{0}(z) e^{Q(z)} f=0,
$$

where $P(z), Q(z)$ are nonconstant polynomials, $A_{1}(z), A_{0}(z)(\not \equiv 0)$ are entire functions such that $\rho\left(A_{1}\right)<\operatorname{deg} P(z), \rho\left(A_{0}\right)<\operatorname{deg} Q(z)$. Gundersen showed in $[7$, p. 419] that if $\operatorname{deg} P(z) \neq \operatorname{deg} Q(z)$, then every nonconstant solution of (1.1) is of infinite order. If $\operatorname{deg} P(z)=\operatorname{deg} Q(z)$, then (1.1) may have nonconstant solutions of finite order. For instance $f(z)=e^{z}+1$ satisfies

2000 Mathematics Subject Classification. 34M10, 30D35.

Key words and phrases. Linear differential equations, meromorphic solutions, order of growth, exponent of convergence of zeros, exponent of convergence of distinct zeros. 
$f^{\prime \prime}+e^{z} f^{\prime}-e^{z} f=0$. In [9], Ki-Ho Kwon has investigated the hyper order of solutions of (1.1) when $\operatorname{deg} P(z)=\operatorname{deg} Q(z)$.

In [4], Z. X. Chen and K. H. Shon have investigated the case when $\operatorname{deg} P(z)=\operatorname{deg} Q(z)$ and have proved the following results:

THEOREM A $([4])$. Let $A_{j}(z)(\neq 0)(j=0,1)$ be meromorphic functions with $\rho\left(A_{j}\right)<1(j=0,1), a, b$ be complex numbers such that $a b \neq 0$ and $\arg a \neq \arg b$ or $a=c b(0<c<1)$. Then every meromorphic solution $f(z) \not \equiv$ 0 of the equation

$$
f^{\prime \prime}+A_{1}(z) e^{a z} f^{\prime}+A_{0}(z) e^{b z} f=0
$$

has infinite order.

In the same paper, Z. X. Chen and K. H. Shon have investigated the fixed points of solutions, their 1st and 2nd derivatives and the differential polynomials and have obtained the following result:

Theorem B ([4]). Let $A_{j}(z)(j=0,1), a, b, c$ satisfy the additional hypotheses of Theorem $A$. Let $d_{0}, d_{1}, d_{2}$ be complex constants that are not all equal to zero. If $f(z) \not \equiv 0$ is any meromorphic solution of equation (1.2), then:

(i) $f, f^{\prime}, f^{\prime \prime}$ all have infinitely many fixed points and satisfy

$$
\bar{\lambda}(f-z)=\bar{\lambda}\left(f^{\prime}-z\right)=\bar{\lambda}\left(f^{\prime \prime}-z\right)=\infty,
$$

(ii) the differential polynomial

$$
g(z)=d_{2} f^{\prime \prime}+d_{1} f^{\prime}+d_{0} f
$$

has infinitely many fixed points and satisfies $\bar{\lambda}(g-z)=\infty$.

Recently Theorem A has been generalized to higher order differential equations by the first named author as follows (see [2]):

Theorem C $([2])$. Let $P_{j}(z)=\sum_{i=0}^{n} a_{i, j} z^{i}(j=0, \ldots, k-1)$ be nonconstant polynomials where $a_{0, j}, \ldots, a_{n, j}(j=0,1, \ldots, k-1)$ are complex numbers such that $a_{n, j} a_{n, 0} \neq 0(j=1, \ldots, k-1)$, let $A_{j}(z)(\not \equiv 0)(j=0, \ldots, k-1)$ be meromorphic functions. Suppose that $\arg a_{n, j} \neq \arg a_{n, 0}$ or $a_{n, j}=c a_{n, 0}$ $(0<c<1)(j=1, \ldots, k-1), \rho\left(A_{j}\right)<n(j=0, \ldots, k-1)$. Then every meromorphic solution $f(z) \not \equiv 0$ of the equation

(1.3) $f^{(k)}+A_{k-1}(z) e^{P_{k-1}(z)} f^{(k-1)}+\ldots+A_{1}(z) e^{P_{1}(z)} f^{\prime}+A_{0}(z) e^{P_{0}(z)} f=0$, where $k \geq 2$, is of infinite order.

The main purpose of this paper is to study the relation between small functions and differential polynomials generated by second order linear differential equation (1.1). For some related results of linear differential equations 
with entire coefficients, we refer the reader to [3]. In fact we will prove the following result:

TheOrem 1.1. Let $P(z)=\sum_{i=0}^{n} a_{i} z^{i}$ and $Q(z)=\sum_{i=0}^{n} b_{i} z^{i}$ be nonconstant polynomials where $a_{i}, b_{i}(i=0,1, \ldots, n)$ are complex numbers, $a_{n} \neq 0, b_{n} \neq 0$ such that $\arg a_{n} \neq \arg b_{n}$ or $a_{n}=c b_{n}(0<c<1)$ and $A_{1}(z), A_{0}(z)(\not \equiv 0)$ be meromorphic functions with $\rho\left(A_{j}\right)<n(j=0,1)$. Let $d_{0}(z), d_{1}(z), d_{2}(z)$ be meromorphic functions that are not all equal to zero with $\rho\left(d_{j}\right)<n$ $(j=0,1,2), \varphi(z) \not \equiv 0$ is a meromorphic function with finite order. If $f(z)$ $\not \equiv 0$ is a meromorphic solution of (1.1), then the differential polynomial $g(z)=d_{2} f^{\prime \prime}+d_{1} f^{\prime}+d_{0} f$ satisfies $\bar{\lambda}(g-\varphi)=\infty$.

REMARK 1.2. Setting $n=1, \varphi(z)=z$ and $d_{0}, d_{1}, d_{2}$ are complex constants that are not all equal to zero in Theorem 1.1, we get Theorem B.

From Theorem 1.1, we obtain the following corollary:

Corollary 1.3. Suppose that $P(z), Q(z), A_{1}(z), A_{0}(z)$ satisfy the hypotheses of Theorem 1.1. If $\varphi(z) \not \equiv 0$ is a meromorphic function with finite order, then every meromorphic solution $f(z) \not \equiv 0$ of (1.1) satisfies $\bar{\lambda}(f-\varphi)=$ $\bar{\lambda}\left(f^{\prime}-\varphi\right)=\bar{\lambda}\left(f^{\prime \prime}-\varphi\right)=\infty$.

\section{Preliminary Lemmas}

We need the following lemmas in the proofs of our theorem.

LEMMA 2.1 ([6]). Let $f$ be a transcendental meromorphic function of finite order $\rho$, let $\Gamma=\left\{\left(k_{1}, j_{1}\right),\left(k_{2}, j_{2}\right), \ldots,\left(k_{m}, j_{m}\right)\right\}$ denote a finite set of distinct pairs of integers that satisfy $k_{i}>j_{i} \geq 0$ for $i=1, \ldots, m$ and let $\varepsilon>0$ be a given constant. Then the following estimations hold:

(i) there exists a set $E_{1} \subset[0,2 \pi)$ that has linear measure zero, such that if $\psi \in[0,2 \pi)-E_{1}$, then there is a constant $R_{1}=R_{1}(\psi)>1$ such that for all $z$ satisfying $\arg z=\psi$ and $|z| \geq R_{1}$ and for all $(k, j) \in \Gamma$, we have

$$
\left|\frac{f^{(k)}(z)}{f^{(j)}(z)}\right| \leq|z|^{(k-j)(\rho-1+\varepsilon)} .
$$

(ii) there exists a set $E_{2} \subset(1, \infty)$ that has finite logarithmic measure, such that for all $z$ satisfying $|z| \notin E_{2} \cup[0,1]$ and for all $(k, j) \in \Gamma$, we have

$$
\left|\frac{f^{(k)}(z)}{f^{(j)}(z)}\right| \leq|z|^{(k-j)(\rho-1+\varepsilon)} .
$$

LEMMA $2.2([1])$. Let $f(z)$ be a transcendental meromorphic function of order $\rho(f)=\rho<+\infty$. Then for any given $\varepsilon>0$, there exists a set $E_{3} \subset[0,2 \pi)$ that has linear measure zero, such that if $\psi_{1} \in[0,2 \pi) \backslash E_{3}$, then 
there is a constant $R_{2}=R_{2}\left(\psi_{1}\right)>1$ such that for all $z$ satisfying $\arg z=\psi_{1}$ and $|z|=r \geq R_{2}$, we have

$$
\exp \left\{-r^{\rho+\varepsilon}\right\} \leq|f(z)| \leq \exp \left\{r^{\rho+\varepsilon}\right\} .
$$

LEMMA 2.3. Let $P(z)=a_{n} z^{n}+\ldots+a_{0},\left(a_{n}=\alpha+i \beta \neq 0\right)$ be a polynomial with degree $n \geq 1$ and $A(z)(\neq 0)$ be a meromorphic function with $\rho(A)<n$. Set $f(z)=A(z) e^{P(z)}, z=r e^{i \theta}, \delta(P, \theta)=\alpha \cos n \theta-\beta \sin n \theta$. Then for any given $\varepsilon>0$, there exists a set $E_{4} \subset[0,2 \pi)$ that has linear measure zero, such that if $\theta \in[0,2 \pi) \backslash\left(E_{4} \cup E_{5}\right)$, where $E_{5}=\{\theta \in[0,2 \pi): \delta(P, \theta)=0\}$ is a finite set, then for sufficiently large $|z|=r$, we have

(i) if $\delta(P, \theta)>0$, then

$$
\exp \left\{(1-\varepsilon) \delta(P, \theta) r^{n}\right\} \leq|f(z)| \leq \exp \left\{(1+\varepsilon) \delta(P, \theta) r^{n}\right\}
$$

(ii) if $\delta(P, \theta)<0$, then

$$
\exp \left\{(1+\varepsilon) \delta(P, \theta) r^{n}\right\} \leq|f(z)| \leq \exp \left\{(1-\varepsilon) \delta(P, \theta) r^{n}\right\} .
$$

Proof. Set $f(z)=h(z) e^{(\alpha+i \beta) z^{n}}$, where $h(z)=A(z) e^{P_{n-1}(z)}$ and $P_{n-1}(z)=P(z)-(\alpha+i \beta) z^{n}$. Then $\rho(h)=\lambda<n$. By Lemma 2.2, for any given $\varepsilon(0<\varepsilon<n-\lambda)$, there is $E_{4} \subset[0,2 \pi)$ that has linear measure zero, such that if $\theta \in[0,2 \pi) \backslash\left(E_{4} \cup E_{5}\right)$, where $E_{5}=\{\theta \in[0,2 \pi): \delta(P, \theta)=0\}$, then there is a constant $R_{2}(\theta)>1$, such that, for all $z$ satisfying $\arg z=\theta$ and $r \geq R_{2}$, we have

$$
\exp \left\{-r^{\lambda+\varepsilon}\right\} \leq|h(z)| \leq \exp \left\{r^{\lambda+\varepsilon}\right\} .
$$

By $\left|e^{(\alpha+i \beta)\left(r e^{i \theta}\right)^{n}}\right|=e^{\delta(P, \theta) r^{n}}$ and (2.6), we have

$$
\exp \left\{\delta(P, \theta) r^{n}-r^{\lambda+\varepsilon}\right\} \leq|f(z)| \leq \exp \left\{\delta(P, \theta) r^{n}+r^{\lambda+\varepsilon}\right\} .
$$

By $\theta \notin E_{5}$ we see that:

(i) if $\delta(P, \theta)>0$, then by $0<\lambda+\varepsilon<n$ and (2.7), we know that (2.4) holds for a sufficiently large $r$;

(ii) if $\delta(P, \theta)<0$, then by $0<\lambda+\varepsilon<n$ and (2.7), we know that (2.5) holds for a sufficiently large $r$.

Lemma $2.4([5])$. Let $A_{0}, A_{1}, \ldots, A_{k-1}, F \neq \equiv 0$ be finite order meromorphic functions. If $f$ is a meromorphic solution with $\rho(f)=\infty$ of the equation

$$
f^{(k)}+A_{k-1} f^{(k-1)}+\ldots+A_{1} f^{\prime}+A_{0} f=F,
$$

then $\bar{\lambda}(f)=\lambda(f)=\rho(f)=\infty$. 
Lemma 2.5. Let $P(z)=\sum_{i=0}^{n} a_{i} z^{i}$ and $Q(z)=\sum_{i=0}^{n} b_{i} z^{i}$ be nonconstant polynomials where $a_{i}, b_{i}(i=0,1, \ldots, n)$ are complex numbers, $a_{n} \neq 0, b_{n} \neq 0$ such that $\arg a_{n} \neq \arg b_{n}$ or $a_{n}=c b_{n}(0<c<1)$. We denote index sets by

$$
\begin{gathered}
\Lambda_{1}=\{0, P\}, \\
\Lambda_{2}=\{0, P, Q, 2 P, P+Q\} .
\end{gathered}
$$

(i) If $H_{j}\left(j \in \Lambda_{1}\right)$ and $H_{Q} \not \equiv 0$ are all meromorphic functions of orders that are less than $n$, setting

$$
\Psi_{1}(z)=\sum_{j \in \Lambda_{1}} H_{j}(z) e^{j}
$$

then $\Psi_{1}(z)+H_{Q} e^{Q} \not \equiv 0$.

(ii) If $H_{j}\left(j \in \Lambda_{2}\right)$ and $H_{2 Q} \neq 0$ are all meromorphic functions of orders that are less than $n$, setting

$$
\Psi_{2}(z)=\sum_{j \in \Lambda_{2}} H_{j}(z) e^{j},
$$

then $\Psi_{2}(z)+H_{2 Q} e^{2 Q} \not \equiv 0$.

Proof. The proof of $(i)$ and $(i i)$ are similar, we prove $(i i)$ only. We divide this into two cases.

CASE 1: Suppose first that $\arg a_{n} \neq \arg b_{n}$. Then $\arg a_{n}, \arg b_{n}$, $\arg \left(a_{n}+b_{n}\right)$ are three distinct arguments. Set $\rho\left(H_{0}\right)=\beta<n$. By Lemma 2.2 , for any given $\varepsilon\left(0<\varepsilon<\min \left(\frac{1}{4}, n-\beta\right)\right)$, there is a set $E_{1}$ that has linear measure zero such that if $\arg z=\theta \in[0,2 \pi) \backslash E_{1}$, then there is $R=R(\theta)>1$ such that for all $z$ satisfying $\arg z=\theta$ and $|z|=r \geq R$, we have

$$
\left|H_{0}(z)\right| \leq \exp \left\{r^{\beta+\varepsilon}\right\} .
$$

By Lemma 2.3, there exists a ray $\arg z=\theta \in[0,2 \pi) \backslash E_{1} \cup E_{2} \cup E_{0}, E_{2}$, $E_{0} \subset[0,2 \pi)$ being defined as in Lemma 2.3, $E_{2}$ having linear measure zero, $E_{0}$ being a finite set, such that

$$
\delta(2 P, \theta)=2 \delta(P, \theta)<0, \delta(P+Q, \theta)<0, \delta(2 Q, \theta)=2 \delta(Q, \theta)>0
$$

and for the above $\varepsilon$, we have for sufficiently large $|z|=r$

$$
\begin{aligned}
& \left|H_{2 Q} e^{2 Q}\right| \geq \exp \left\{(1-\varepsilon) 2 \delta(Q, \theta) r^{n}\right\}, \\
& \left|H_{Q} e^{Q}\right| \leq \exp \left\{(1+\varepsilon) \delta(Q, \theta) r^{n}\right\}, \\
& \left|H_{P+Q} e^{P+Q}\right| \leq \exp \left\{(1-\varepsilon) \delta(P+Q, \theta) r^{n}\right\}<1, \\
& \left|H_{2 P} e^{2 P}\right| \leq \exp \left\{(1-\varepsilon) 2 \delta(P, \theta) r^{n}\right\}<1, \\
& \left|H_{P} e^{P}\right| \leq \exp \left\{(1-\varepsilon) \delta(P, \theta) r^{n}\right\}<1 .
\end{aligned}
$$


If $\Psi_{2}(z)+H_{2 Q} e^{2 Q} \equiv 0$, then by $(2.9)-(2.14)$, we have

$$
\begin{aligned}
\exp \{ & \left.(1-\varepsilon) 2 \delta(Q, \theta) r^{n}\right\} \\
& \leq\left|H_{2 Q} e^{2 Q}\right| \leq \exp \left\{r^{\beta+\varepsilon}\right\}+\exp \left\{(1+\varepsilon) \delta(Q, \theta) r^{n}\right\}+3 \\
& \leq 3 \exp \left\{r^{\beta+\varepsilon}\right\} \exp \left\{(1+\varepsilon) \delta(Q, \theta) r^{n}\right\} .
\end{aligned}
$$

By $2(1-\varepsilon)-(1+\varepsilon)=1-3 \varepsilon>\frac{1}{4}$, we have

$$
\exp \left\{\frac{1}{4} \delta(Q, \theta) r^{n}\right\} \leq 3 \exp \left\{r^{\beta+\varepsilon}\right\} .
$$

This is a contradiction by $\beta+\varepsilon<n$. Hence $\Psi_{2}(z)+H_{2 Q} e^{2 Q} \not \equiv 0$.

CAse 2: Suppose now $a_{n}=c b_{n}(0<c<1)$. Then for any $\operatorname{ray} \arg z=\theta$, we have

$$
\begin{gathered}
\delta(P, \theta)=c \delta(Q, \theta), \quad \delta(2 P, \theta)=2 c \delta(Q, \theta), \\
\delta(P+Q, \theta)=(1+c) \delta(Q, \theta), \quad \delta(2 Q, \theta)=2 \delta(Q, \theta) .
\end{gathered}
$$

Then by Lemma 2.2 and Lemma 2.3 , for any given $\varepsilon\left(0<\varepsilon<\min \left\{\frac{1-c}{4}, n-\beta\right\}\right)$ there exist $E_{j} \subset[0,2 \pi)(j=0,1,2)$ that have linear measure zero, where $E_{0}, E_{1}$ and $E_{2}$ are defined as in the case 1 respectively. We take the ray $\arg z=\theta \in[0,2 \pi) \backslash E_{1} \cup E_{2} \cup E_{0}$ such that $\delta(Q, \theta)>0$ and for sufficiently large $|z|=r$, we have $(2.9)-(2.11)$ and

$$
\begin{aligned}
& \left|H_{P} e^{P}\right| \leq \exp \left\{(1+\varepsilon) c \delta(Q, \theta) r^{n}\right\}, \\
& \left|H_{P+Q} e^{P+Q}\right| \leq \exp \left\{(1+\varepsilon)(1+c) \delta(Q, \theta) r^{n}\right\}, \\
& \left|H_{2 P} e^{2 P}\right| \leq \exp \left\{(1+\varepsilon) 2 c \delta(Q, \theta) r^{n}\right\} .
\end{aligned}
$$

If $\Psi_{2}(z)+H_{2 Q} e^{2 Q} \equiv 0$, then by $(2.9)-(2.11)$ and $(2.17)-(2.19)$ we have

$$
\exp \left\{(1-\varepsilon) 2 \delta(Q, \theta) r^{n}\right\} \leq\left|H_{2 Q} e^{2 Q}\right| \leq \exp \left\{r^{\beta+\varepsilon}\right\}
$$

$$
+2 \exp \left\{(1+\varepsilon)(1+c) \delta(Q, \theta) r^{n}\right\}+2 \exp \left\{(1+\varepsilon) 2 c \delta(Q, \theta) r^{n}\right\} .
$$

By $\beta+\varepsilon<n$ and $4 \varepsilon<1-c$, we have, as $r \rightarrow+\infty$

$$
\begin{aligned}
& \frac{\exp \left\{r^{\beta+\varepsilon}\right\}}{\exp \left\{(1-\varepsilon) 2 \delta(Q, \theta) r^{n}\right\}} \rightarrow 0, \\
& \frac{\exp \left\{(1+\varepsilon)(1+c) \delta(Q, \theta) r^{n}\right\}}{\exp \left\{(1-\varepsilon) 2 \delta(Q, \theta) r^{n}\right\}} \rightarrow 0, \\
& \frac{\exp \left\{(1+\varepsilon) 2 c \delta(Q, \theta) r^{n}\right\}}{\exp \left\{(1-\varepsilon) 2 \delta(Q, \theta) r^{n}\right\}} \rightarrow 0 .
\end{aligned}
$$

By $(2.20)-(2.23)$, we get $1 \leq 0$. This is a contradiction, hence $\Psi_{2}(z)+H_{2 Q} e^{2 Q}$ $\not \equiv 0$. 
Lemma 2.6. Let $\Psi_{20}(z), \Psi_{21}(z), \Psi_{22}(z), \Psi_{23}(z), \Psi_{24}(z)$ have the form of $\Psi_{2}(z)$ which is defined as in Lemma 2.5 (ii) and $H_{2 Q} \neq \equiv$ is a meromorphic function of order $\rho\left(H_{2 Q}\right)<n, \varphi(z) \neq \equiv$ is a meromorphic function with finite order. Then every meromorphic solution $w \neq \equiv$ of the following equation

$$
\begin{aligned}
\Psi_{24}(z) w^{\prime \prime} & +\left(\Psi_{23}(z)+\frac{\varphi^{\prime}(z)}{\varphi(z)} \Psi_{22}(z)\right) w^{\prime} \\
& +\left(\frac{\varphi^{\prime}(z)}{\varphi(z)} \Psi_{21}(z)+\Psi_{20}(z)+H_{2 Q} e^{2 Q}\right) w=0
\end{aligned}
$$

is of infinite order.

Proof. Suppose that $w \neq \equiv 0$ is a meromorphic solution of (2.24) with $\rho(w)<\infty$. Set $\rho=\max \{\rho(w), \rho(\varphi)\}<\infty$. Then by Lemma 2.1, for any given $\varepsilon>0$, there exists a set $E \subset[0,2 \pi)$ that has linear measure zero, such that if $\theta \in[0,2 \pi)-E$, then there is a constant $R=R(\theta)>1$ such that for all $z$ satisfying $\arg z=\theta$ and $|z| \geq R$, we have

$$
\left|\frac{w^{(k)}(z)}{w(z)}\right| \leq|z|^{k(\rho-1+\varepsilon)}, \quad\left|\frac{\varphi^{(k)}(z)}{\varphi(z)}\right| \leq|z|^{k(\rho-1+\varepsilon)} \quad(k=1,2) .
$$

It follows that on the ray $\arg z=\theta \in[0,2 \pi)-E$,

$$
\begin{gathered}
\frac{w^{(k)}(z)}{w(z)} H_{j}(z) e^{j} \quad\left(k=1,2 ; j \in \Lambda_{2}\right), \\
\frac{\varphi^{\prime}(z)}{\varphi(z)} \frac{w^{\prime}(z)}{w(z)} H_{j}(z) e^{j} \quad\left(j \in \Lambda_{2}\right), \frac{\varphi^{\prime}(z)}{\varphi(z)} H_{j}(z) e^{j} \quad\left(j \in \Lambda_{2}\right)
\end{gathered}
$$

keep the properties of $H_{j}(z) e^{j}\left(j \in \Lambda_{2}\right)$ which are defined as in (2.9), (2.11)$(2.14)$ or $(2.9),(2.11),(2.17)-(2.19)$. By using similar reasoning to that in the proof of Lemma $2.5(i i)$, we obtain a contradiction. Then $\rho(w)=\infty$.

\section{Proof of Theorem}

We first prove $\rho(g)=\rho\left(d_{2} f^{\prime \prime}+d_{1} f^{\prime}+d_{0} f\right)=\infty$. Suppose that $f(z) \not \equiv$ 0 is a meromorphic solution of equation (1.1). Then by Theorem $\mathrm{C}$ we have $\rho(f)=\infty$. First we suppose that $d_{2}(z) \not \equiv 0$. Substituting $f^{\prime \prime}=-A_{1} e^{P} f^{\prime}-$ $A_{0} e^{Q} f$ into $g$, we get

$$
g=\left(d_{1}-d_{2} A_{1} e^{P}\right) f^{\prime}+\left(d_{0}-d_{2} A_{0} e^{Q}\right) f .
$$

Differentiating both sides of equation (3.1) and replacing $f^{\prime \prime}$ with $f^{\prime \prime}=$ $-A_{1} e^{P} f^{\prime}-A_{0} e^{Q} f$, we obtain

$$
\begin{aligned}
& g^{\prime}= {\left[d_{2} A_{1}^{2} e^{2 P}-\left(\left(d_{2} A_{1}\right)^{\prime}+P^{\prime} d_{2} A_{1}+d_{1} A_{1}\right) e^{P}-d_{2} A_{0} e^{Q}+d_{0}+d_{1}^{\prime}\right] f^{\prime} } \\
&(3.2) \quad+\left[d_{2} A_{0} A_{1} e^{P+Q}-\left(\left(d_{2} A_{0}\right)^{\prime}+Q^{\prime} d_{2} A_{0}+d_{1} A_{0}\right) e^{Q}+d_{0}^{\prime}\right] f .
\end{aligned}
$$


Set

$$
\begin{aligned}
\alpha_{1}= & d_{1}-d_{2} A_{1} e^{P}, \quad \alpha_{0}=d_{0}-d_{2} A_{0} e^{Q} \\
\beta_{1}= & \alpha_{1}^{\prime}+\alpha_{0}-\alpha_{1} A_{1} e^{P} \\
= & d_{2} A_{1}^{2} e^{2 P}-\left(\left(d_{2} A_{1}\right)^{\prime}+P^{\prime} d_{2} A_{1}+d_{1} A_{1}\right) e^{P} \\
& -d_{2} A_{0} e^{Q}+d_{0}+d_{1}^{\prime}, \\
\beta_{0}= & \alpha_{0}^{\prime}-\alpha_{1} A_{0} e^{Q}=d_{2} A_{0} A_{1} e^{P+Q} \\
& -\left(\left(d_{2} A_{0}\right)^{\prime}+Q^{\prime} d_{2} A_{0}+d_{1} A_{0}\right) e^{Q}+d_{0}^{\prime} .
\end{aligned}
$$

Then we have

$$
\begin{aligned}
& \alpha_{1} f^{\prime}+\alpha_{0} f=g, \\
& \beta_{1} f^{\prime}+\beta_{0} f=g^{\prime} .
\end{aligned}
$$

Set

$$
\begin{aligned}
h= & \alpha_{1} \beta_{0}-\alpha_{0} \beta_{1} \\
= & \left(d_{1}-d_{2} A_{1} e^{P}\right)\left[d_{2} A_{0} A_{1} e^{P+Q}-\left(\left(d_{2} A_{0}\right)^{\prime}+Q^{\prime} d_{2} A_{0}+d_{1} A_{0}\right) e^{Q}+d_{0}^{\prime}\right] \\
& -\left(d_{0}-d_{2} A_{0} e^{Q}\right)\left[d_{2} A_{1}^{2} e^{2 P}-\left(\left(d_{2} A_{1}\right)^{\prime}+P^{\prime} d_{2} A_{1}+d_{1} A_{1}\right) e^{P}\right. \\
(3.8) \quad & \left.-d_{2} A_{0} e^{Q}+d_{0}+d_{1}^{\prime}\right] .
\end{aligned}
$$

Now check all the terms of $h$. Since the term $d_{2}^{2} A_{1}^{2} A_{0} e^{2 P+Q}$ is eliminated, by (3.8) we can write $h=\Psi_{2}(z)-d_{2}^{2} A_{0}^{2} e^{2 Q}$, where $\Psi_{2}(z)$ is defined as in Lemma 2.5 (ii). By $d_{2} \not \equiv 0, A_{0} \not \equiv 0$ and Lemma 2.5 (ii) we see that $h \not \equiv 0$. By (3.6), (3.7), we obtain

$$
\begin{aligned}
h f & =\alpha_{1} g^{\prime}-\beta_{1} g, \\
h f^{\prime} & =-\alpha_{0} g^{\prime}+\beta_{0} g .
\end{aligned}
$$

Differentiating both sides of equation (3.10) we obtain

$$
\left(h f^{\prime}\right)^{\prime}=-\alpha_{0} g^{\prime \prime}+\left(\beta_{0}-\alpha_{0}^{\prime}\right) g^{\prime}+\beta_{0}^{\prime} g .
$$

On the other hand by (1.1), (3.9) and (3.10)

$$
\begin{aligned}
\left(h f^{\prime}\right)^{\prime} & =h^{\prime} f^{\prime}+h f^{\prime \prime}=\left(h^{\prime}-h A_{1} e^{P}\right) f^{\prime}-h A_{0} e^{Q} f \\
& =\left(\frac{h^{\prime}}{h}-A_{1} e^{P}\right)\left(-\alpha_{0} g^{\prime}+\beta_{0} g\right)-A_{0} e^{Q}\left(\alpha_{1} g^{\prime}-\beta_{1} g\right) .
\end{aligned}
$$

Equating (3.11), (3.12) and applying (3.5) yields

(3.13) $\alpha_{0} g^{\prime \prime}-\alpha_{0}\left(\frac{h^{\prime}}{h}-A_{1} e^{P}\right) g^{\prime}+\left[\beta_{0}\left(\frac{h^{\prime}}{h}-A_{1} e^{P}\right)+\beta_{1} A_{0} e^{Q}-\beta_{0}^{\prime}\right] g=0$. 
Hence by $(3.3),(3.4),(3.5)$, we can write (3.13) in the form

$$
\begin{aligned}
\Psi_{24}(z) g^{\prime \prime} & +\left(\Psi_{23}(z)+\frac{h^{\prime}(z)}{h(z)} \Psi_{22}(z)\right) g^{\prime} \\
& +\left(\frac{h^{\prime}(z)}{h(z)} \Psi_{21}(z)+\Psi_{20}(z)+H_{2 Q} e^{2 Q}\right) g=0,
\end{aligned}
$$

where

$$
\begin{aligned}
& \Psi_{24}(z)=\alpha_{0}=d_{0}-d_{2} A_{0} e^{Q}, \\
& \Psi_{23}(z)=A_{1} e^{P} \alpha_{0}=d_{0} A_{1} e^{P}-d_{2} A_{0} A_{1} e^{P+Q}, \\
& \begin{aligned}
\Psi_{22}(z) & =-\alpha_{0}=d_{2} A_{0} e^{Q}-d_{0}, \\
\Psi_{21}(z) & =\beta_{0}=d_{2} A_{0} A_{1} e^{P+Q}-\left(\left(d_{2} A_{0}\right)^{\prime}+Q^{\prime} d_{2} A_{0}+d_{1} A_{0}\right) e^{Q}+d_{0}^{\prime}, \\
\Psi_{20}(z) & +H_{2 Q} e^{2 Q}=\beta_{1} A_{0} e^{Q}-\beta_{0} A_{1} e^{P}-\beta_{0}^{\prime} \\
= & \left(\alpha_{1}^{\prime}+\alpha_{0}-\alpha_{1} A_{1} e^{P}\right) A_{0} e^{Q}-\left(\alpha_{0}^{\prime}-\alpha_{1} A_{0} e^{Q}\right) A_{1} e^{P}-\beta_{0}^{\prime} \\
= & \alpha_{1}^{\prime} A_{0} e^{Q}+\alpha_{0} A_{0} e^{Q}-\alpha_{0}^{\prime} A_{1} e^{P}-\beta_{0}^{\prime} \\
= & \alpha_{1}^{\prime} A_{0} e^{Q}+\left(d_{0}-d_{2} A_{0} e^{Q}\right) A_{0} e^{Q}-\alpha_{0}^{\prime} A_{1} e^{P}-\beta_{0}^{\prime} \\
= & \alpha_{1}^{\prime} A_{0} e^{Q}+d_{0} A_{0} e^{Q}-\alpha_{0}^{\prime} A_{1} e^{P}-\beta_{0}^{\prime}-d_{2} A_{0}^{2} e^{2 Q} .
\end{aligned}
\end{aligned}
$$

But $\rho(h) \leq n, \Psi_{24} \not \equiv 0, d_{2} A_{0}^{2} \not \equiv 0$ and by Lemma 2.6 , we obtain $\rho(g)=\infty$.

If $d_{2} \equiv 0, d_{1} \not \equiv 0$, then $g=d_{1} f^{\prime}+d_{0} f$ and $g^{\prime}=d_{1} f^{\prime \prime}+\left(d_{1}^{\prime}+d_{0}\right) f^{\prime}+d_{0}^{\prime} f$. Since $d_{1} \not \equiv 0$, we conclude $\rho\left(g^{\prime}\right)=\infty$ by using a similar argument as for the case $d_{2} \not \equiv 0$. Thus $\rho(g)=\infty$.

Now we prove $\bar{\lambda}(g-\varphi)=\infty$. First we suppose that $d_{2}(z) \not \equiv 0$. Set $w=g-\varphi$, then $\rho(w)=\infty, \bar{\lambda}(w)=\bar{\lambda}(g-\varphi)$. Substituting $g=w+\varphi$, $g^{\prime}=w^{\prime}+\varphi^{\prime}, g^{\prime \prime}=w^{\prime \prime}+\varphi^{\prime \prime}$ into (3.14), we obtain

$$
\begin{aligned}
\Psi_{24}(z) w^{\prime \prime}+ & \left(\Psi_{23}(z)+\frac{h^{\prime}(z)}{h(z)} \Psi_{22}(z)\right) w^{\prime} \\
& +\left(\frac{h^{\prime}(z)}{h(z)} \Psi_{21}(z)+\Psi_{20}(z)+H_{2 Q} e^{2 Q}\right) w \\
= & -\left[\Psi_{24}(z) \varphi^{\prime \prime}+\left(\Psi_{23}(z)+\frac{h^{\prime}(z)}{h(z)} \Psi_{22}(z)\right) \varphi^{\prime}\right. \\
& \left.+\left(\frac{h^{\prime}(z)}{h(z)} \Psi_{21}(z)+\Psi_{20}(z)+H_{2 Q} e^{2 Q}\right) \varphi\right] .
\end{aligned}
$$

Since $\varphi(z) \not \equiv 0$ is a finite order meromorphic function, Lemma 2.6 implies

$$
\begin{aligned}
\Psi_{24}(z) \varphi^{\prime \prime} & +\left(\Psi_{23}(z)+\frac{h^{\prime}(z)}{h(z)} \Psi_{22}(z)\right) \varphi^{\prime} \\
& +\left(\frac{h^{\prime}(z)}{h(z)} \Psi_{21}(z)+\Psi_{20}(z)+H_{2 Q} e^{2 Q}\right) \varphi \neq \equiv .
\end{aligned}
$$


Hence by Lemma 2.4, we $\bar{\lambda}(w)=\lambda(w)=\rho(w)=\infty$. Then $\bar{\lambda}(g-\varphi)=\infty$.

Suppose $d_{2} \equiv 0, d_{1} \neq 0$. Using a similar reasoning as above we get $\bar{\lambda}(w)=\rho(w)=\infty$.

Finally, if $d_{2} \equiv 0, d_{1} \equiv 0, d_{0} \not \equiv 0$ then we have $w=d_{0} f-\varphi, \rho(w)=\infty$. By substituting

$$
f=\frac{w}{d_{0}}+\frac{\varphi}{d_{0}}, \quad f^{\prime}=\left(\frac{w}{d_{0}}\right)^{\prime}+\left(\frac{\varphi}{d_{0}}\right)^{\prime}, f^{\prime \prime}=\left(\frac{w}{d_{0}}\right)^{\prime \prime}+\left(\frac{\varphi}{d_{0}}\right)^{\prime \prime}
$$

into equation (1.1) we obtain

$$
\left(\frac{w}{d_{0}}\right)^{\prime \prime}+A_{1} e^{P}\left(\frac{w}{d_{0}}\right)^{\prime}+A_{0} e^{Q} \frac{w}{d_{0}}=-\left(\left(\frac{\varphi}{d_{0}}\right)^{\prime \prime}+A_{1} e^{P}\left(\frac{\varphi}{d_{0}}\right)^{\prime}+A_{0} e^{Q} \frac{\varphi}{d_{0}}\right) .
$$

Writing (3.17) in the form

$$
w^{\prime \prime}+\Phi_{1} w^{\prime}+\Phi_{0} w=-d_{0}\left(\left(\frac{\varphi}{d_{0}}\right)^{\prime \prime}+A_{1} e^{P}\left(\frac{\varphi}{d_{0}}\right)^{\prime}+A_{0} e^{Q} \frac{\varphi}{d_{0}}\right)
$$

where $\Phi_{1}(z)$ and $\Phi_{0}(z)$ are meromorphic functions with $\rho\left(\Phi_{1}\right) \leq n, \rho\left(\Phi_{0}\right) \leq$ $n$. Since $d_{0} \not \equiv 0$ and $\frac{\varphi(z)}{d_{0}(z)}$ is a finite order meromorphic function so by Theorem $\mathrm{C}$ we have

$$
d_{0}\left(\left(\frac{\varphi}{d_{0}}\right)^{\prime \prime}+A_{1} e^{P}\left(\frac{\varphi}{d_{0}}\right)^{\prime}+A_{0} e^{Q} \frac{\varphi}{d_{0}}\right) \not \equiv 0 .
$$

Hence by Lemma 2.4, we have $\bar{\lambda}(w)=\rho(w)=\infty$. Then $\bar{\lambda}(w)=\infty$, i.e., $\bar{\lambda}\left(d_{0} f-\varphi\right)=\infty$.

ACKNOWLEDGEMENTS.

The authors would like to thank the referee for his/her helpful remarks and suggestions.

\section{REFERENCES}

[1] S. B. Bank and J. K. Langley, On the oscillation of solutions of certain linear differential equations in the complex domain, Proc. Edinburgh Math. Soc. (2) 30 (1987), 455-469.

[2] B. Belaïdi, On the meromorphic solutions of linear differential equations, J. Syst. Sci. Complex. 20 (2007) 41-46.

[3] Z. X. Chen, K. H. Shon, The relation between solutions of a class of second order differential equations with functions of small growth, Chinese Ann. Math. Ser. A 27 (2006), 431-442 (in Chinese).

[4] Z. X. Chen, K. H. Shon, On the growth and fixed points of solutions of second order differential equations with meromorphic coefficients, Acta Math. Sin. (Engl. Ser.) 21 (2005), 753-764.

[5] Z. X. Chen, Zeros of meromorphic solutions of higher order linear differential equations, Analysis 14 (1994), 425-438.

[6] G. G. Gundersen, Estimates for the logarithmic derivative of a meromorphic function, plus similar estimates, J. London Math. Soc. (2) 37 (1988), 88-104. 
[7] G. G. Gundersen, Finite order solutions of second order linear differential equations, Trans. Amer. Math. Soc. 305 (1988), 415-429.

[8] W. K. Hayman, Meromorphic functions, Clarendon Press, Oxford, 1964.

[9] K. H. Kwon, Nonexistence of finite order solutions of certain second order linear differential equations, Kodai Math. J. 19 (1996), 378-387.

[10] R. Nevanlinna, Eindeutige Analytische Funktionen, Zweite Auflage. Reprint. Die Grundlehren der mathematischen Wissenschaften, Band 46. Springer-Verlag, BerlinNew York, 1974.

\section{A. El Farissi}

Department of Mathematics

Laboratory of Pure and Applied Mathematics

University of Mostaganem

B. P 227 Mostaganem

Algeria

B. Belaïdi

Department of Mathematics

Laboratory of Pure and Applied Mathematics

University of Mostaganem

B. P 227 Mostaganem

Algeria

E-mail: belaidi@univ-mosta.dz \& belaidi.benharrat@caramail.com

Received: 11.5.2007.

Revised: 1.2.2008. 